\title{
"Triple-Alls" Distribution Network Information Model and Platform Design
}

\author{
Jianfeng Wang ${ }^{1}$, Liang Chen ${ }^{1}$, Liqin Shi ${ }^{2}$, Dao 'an Zhang ${ }^{3}$, and Hao Liu ${ }^{1}$ \\ ${ }^{1}$ State Grid Zhejiang Electric Power Co., Ltd. Huzhou Power Supply Company, Huzhou, Zhejiang \\ ${ }^{2}$ Hangzhou WR Science and Technology Co., Ltd.,Hangzhou, Zhejiang \\ ${ }^{3}$ Huzhou Electric Power Design Institute, Huzhou, Zhejiang
}

\begin{abstract}
In recent years, large-scale new energy sources have been connected to the power distribution network, and the ubiquitous power IoT sensing technology has developed rapidly. At the same time, the increase in energy consumption has placed higher requirements on the consumption of new energy, which has led to the "Triple-Alls" (All access, All sensing, All consumption) requirements of the distribution network. To this end, this paper conducts a series of studies on the "Triple-Alls" distribution network: First, it analyzes the characteristics and key technologies of the "Triple-Alls" distribution network by comparing with traditional distribution networks, and then establishes energy storage, photovoltaic, and wind power. Information model of "Triple-Alls" distribution network. Then, a platform design scheme meeting the requirements is constructed. Finally, the application functions of "All access", "All sensing" and "All consumption" in the distribution network are explained in detail.
\end{abstract}

\section{Introduction}

The distribution network is located at the end of the power system's power supply, playing a role of directly connecting the power system with the user. In recent years, with the large-scale access of electric vehicles, distributed energy, microgrid, energy storage devices and other facilities, as well as the opening of the power market and the emergence of various power needs, it is required that all consumption of clean energy, All access to multiple loads, and equipment. The important content of the research on distribution network with the "TripleAlls" feature.

The "Triple-Alls" distribution network connects all power generation power to the grid through clean energy access planning, and consumes all the power generated by clean energy. It also all senses the entire distribution network through advanced measurement and Internet of things sensing technology.

Many experts and scholars have studied the design of traditional distribution network information models and operating platforms. Reference [1] designed the interactive interface of the electricity consumption information acquisition system for smart electricity related business platforms such as 95598 service, energy service management platform, intelligent building and intelligent community system, and marketing business application system, and proposed an existing platform architecture. Information [2] and [3] proposed the intelligent power distribution communication network architecture for the ubiquitous electric power Internet of Things; [4] designed for the data center system, including: source layer (basic Data), sharing layer (public data and extracted data), and analysis layer (providing model services). The three-tier service architecture proposes the key technologies required for the construction of the data center. Reference [5] starts from the construction of the ubiquitous electric power Internet of Things. The goal is to study the network structure of the distribution network based on its specific scenario. In the context of the energy Internet, a new ubiquitous power IoT distribution network topology structure is proposed, which uses new equipment of energy routers. Literature [6] proposed the optimal evaluation index system of intelligent power distribution cloud platform based on improved interval analytic method for the optimal evaluation of intelligent power distribution cloud platform.

In the research of power grid information model, [7] analyzed the development status of power grid model, expounded the power grid public information model and its component interface interaction specifications, analyzed the extension principles and methods, and expounded the CIM model in intelligent power distribution. Developments in power grids and microgrids. Reference [8] builds a unified information model on the basis of China Southern Power Grid, and realizes the unification of the main network model, the distribution network model, and the customer model, including the physical unified model and the logical unified model. Aiming at the research on new energy and new load models, [9], in order to meet the model requirements of active power distribution networks, extended the CIM model guidelines and established a 
DC microgrid CIM model for new energy. Literature [10] researched the electricity consumption behavior of air conditioners in the aspect of new electricity load, expounded its main electricity components and electricity cycle characteristics, and analyzed the principle of air conditioning refrigeration. No corresponding information model was established.

Analysis of the above literatures reveals that, although many literatures have done a lot of in-depth research on the traditional distribution network information models and architectures, there have been few reports on the research of "Triple-Alls" distribution networks. Therefore, this article conducts relevant research on the "Triple-Alls" distribution network. Firstly, it analyzes its characteristics and key technologies. Then it describes the information model of the "Triple-Alls" distribution network and builds a "Triple-Alls" distribution network. The platform architecture finally elaborates the implementation of the "Triple-Alls" distribution network's "All access", "All sensing" and "All consumption" application functional modules.

\section{2 "Triple-alls" distribution network features and key technologies}

With the widespread access to new energy sources and the application of virtual power plant technology, the "Triple-Alls" distribution network presents very different characteristics from the traditional distribution network. The specific performance is as follows.

\subsection{Features of "triple-alls" distribution network}

\subsubsection{The power generation and load are diversified, and the importance of comprehensive and coordinated dispatch is prominent}

The "Triple-Alls" distribution network contains a large number of distributed energy sources. Large-scale applications of distributed energy sources such as wind power and photovoltaics are affected by the external environment such as weather and geography, and present strong uncertainty and randomness. There are various loads in the power grid. There is a large difference in the power consumption characteristics of electric vehicles, electric heating, and port and shore power. In order to realize the real-time balance of power generation and load, it is necessary to realize the comprehensive coordination and dispatching of the "Triple-Alls" distribution network.

\subsubsection{Terminals of huge devices are integrated into one, and there are problems of multi-source data fusion, mutual communication, and information sharing}

Due to the All access of multiple power sources and user terminals of the "Triple-Alls" distribution network, the huge equipment terminals are connected into one. The new generation distribution network has different characteristics of multi-source data types and different update times. In addition, the real-time communication of multiple terminal devices has become the key to the new generation of "Triple-Alls" distribution network.

\subsubsection{Clean and intelligent energy consumption}

In the distribution network, electric vehicles, electricity heating and cooling, etc. will realize the direct replacement of fossil energy by clean energy electricity at the terminal, and the proportion of electric energy in the terminal consumption will continue to rise. The construction of smart communities and smart homes enables customers to be more involved in power management, thereby developing ladder electricity prices, real-time electricity prices, and precise load control.

\subsection{Key technologies of new generation "three all" distribution networks}

\subsubsection{Ubiquitous internet of things technology}

"Three All" distribution network system interconnects all things in all links, its wide coverage, equipment types, environmental factors directly related to it are also very rich, almost all kinds of sensors can play a role in the system. Advanced situational awareness technology can provide good data resources for coordinated and optimized scheduling of multiple energy sources, and can solve a series of issues such as "what is happening in the environment", "why will happen" and "what will happen". In addition, the new generation "Triple-Alls" distribution network must not only realize the perception, collection, and transmission of various state data, but also effectively integrate, store, and compress the massive and messy raw data to improve the operating efficiency of the power grid [11].

\subsubsection{Advanced communication technology}

The "Triple-Alls" distribution network will adopt advanced, reliable, stable, and efficient emerging communication technologies and systems to enrich the communication access methods of the distribution network, transforming from simply satisfying business needs to passively satisfying business needs to being actively leading.

5G technology provides a solution for the "last kilometer" wireless access communication coverage of the power distribution communication network. Services such as low-voltage collection and distributed energy access in the "Triple-Alls" power distribution network can make breakthroughs with the help of 5G. 5G networks can take advantage of its ultra-high bandwidth, ultra-low time delay, and ultra-large-scale connections to improve the "Triple-Alls" distribution network's ability to control its own business.

\subsubsection{Virtual power plant technology}


Virtual Power Plant (VPP) integrates various distributed power sources through sophisticated control methods and energy management, packs multiple small power sources, and outputs relatively stable and large output. It aggregates multiple distributed energy sources to achieve the stability and reliability of the overall output, and provides efficient power for the "Triple-Alls" power grid, thereby ensuring the stability and security of its grid connection [12].

\subsubsection{Cloud computing technology}

Cloud computing technology is a key technology to realize the efficient storage, reading and processing of massive information data in the ubiquitous electric power Internet of Things, and is an important part of the data layer. Through the cloud platform technology, the "Three All" distribution network resources can be reasonably configured and called, so as to achieve the purpose of efficient use of different computing resources.

\section{3 "Triple-alls" distribution network information model}

The information model of the "Triple-Alls" distribution network includes logical models and physical models. This article focuses on the logical model of the "TripleAlls" distribution network, as shown in Fig1.

Based on the traditional distribution network model, the power generation model, load model and measurement model are extended. The main changes are shown below. The energy storage model and electric vehicle are taken as examples to illustrate the extended CIM model.

\subsection{Extended power generation model}

In addition to the traditional power generation model, energy storage models, wind farm models, and photovoltaic power plant models have been added $[8,13]$.

Energy storage model: Describes the energy storage unit, which depends on and extends the power generation package of the traditional model, including sub-models such as battery power stations and super capacitors. This model can meet the characteristics of energy input and output bidirectional scheduling. The battery energy storage power plant model (as shown in Fig 2, the solid line frame in the figure represents the existing class, and the dashed line represents the extended class) rely on and extend the power system resource package in the traditional model. In order to realize the economic dispatch of the battery energy storage unit, the model uses a class of charge and discharge planning.

Wind farm model: This model relies on and extends the power system resource package in the traditional model. This model derives two sub-models of offshore wind farm and onshore wind farm. Wind turbines include synchronous generators and asynchronous generators, so the wind turbine class can be clustered with synchronous generators and asynchronous generators $[14,15]$.

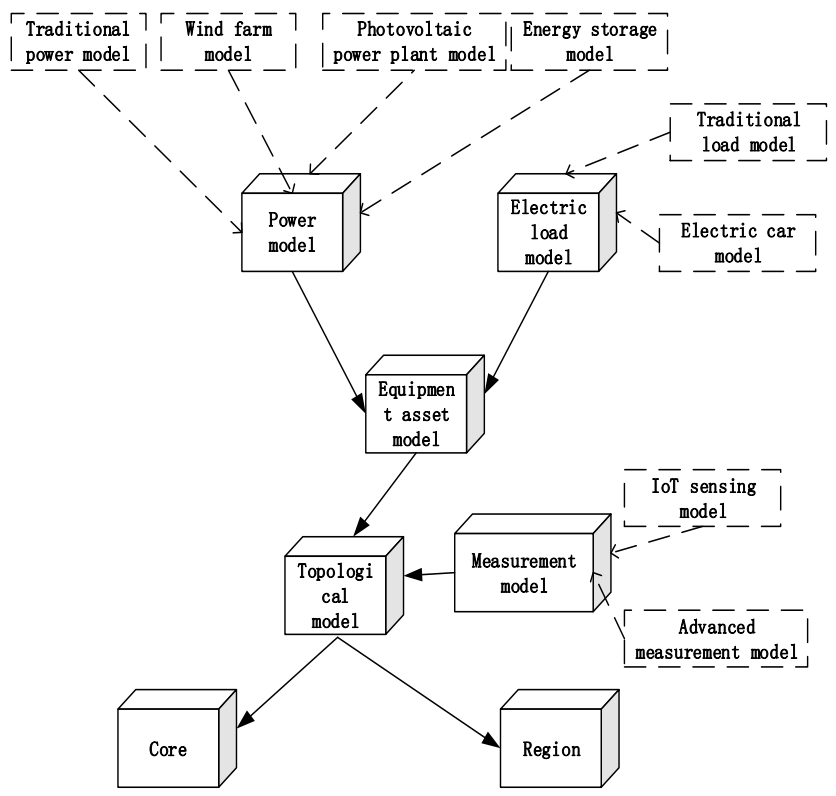

Fig. 1. Schematic diagram of "Triple-Alls" distribution network information logic model

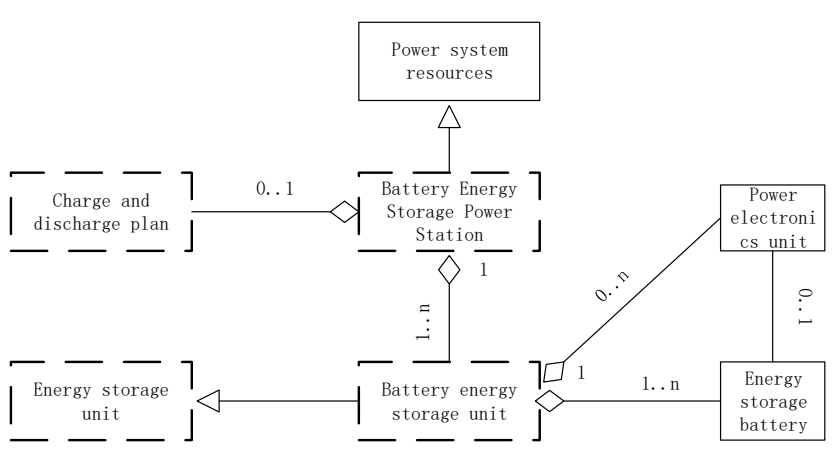

Fig. 2. CIM Model of Battery Energy Storage Power Station

Photovoltaic power plant model: Photovoltaic panels directly convert solar energy into DC power, which is a prime mover. Photovoltaic arrays are formed by series and parallel photovoltaic modules, so photovoltaic arrays are derived from prime movers and have a one-to-many aggregation relationship with photovoltaic modules.

\subsection{Expansion model of electric load}

The electric vehicle model that has a greater impact on the "Triple-Alls" distribution network is added, as shown in Fig 4.

The electric vehicle model inherits the power system resources of the traditional distribution network information model. Considering that the important equipment of the electric vehicle charging station is the charging pile and the off-board charger, the model extends the electric vehicle charging pile model and the off-board charger sub-model. 


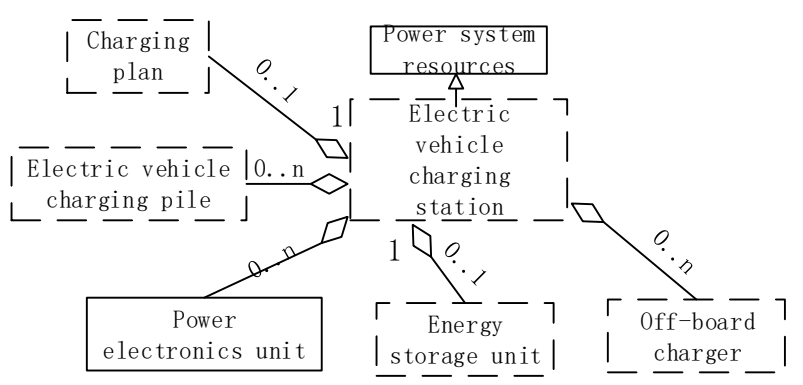

Fig. 3. CIM model of electric vehicle charging pile

\section{4 "Triple-alls" distribution network platform architecture design}

"Triple-Alls" distribution network service platform includes four parts from bottom to top: perception layer, network layer, platform layer and application layer. The overall design scheme is shown in Fig. 4.

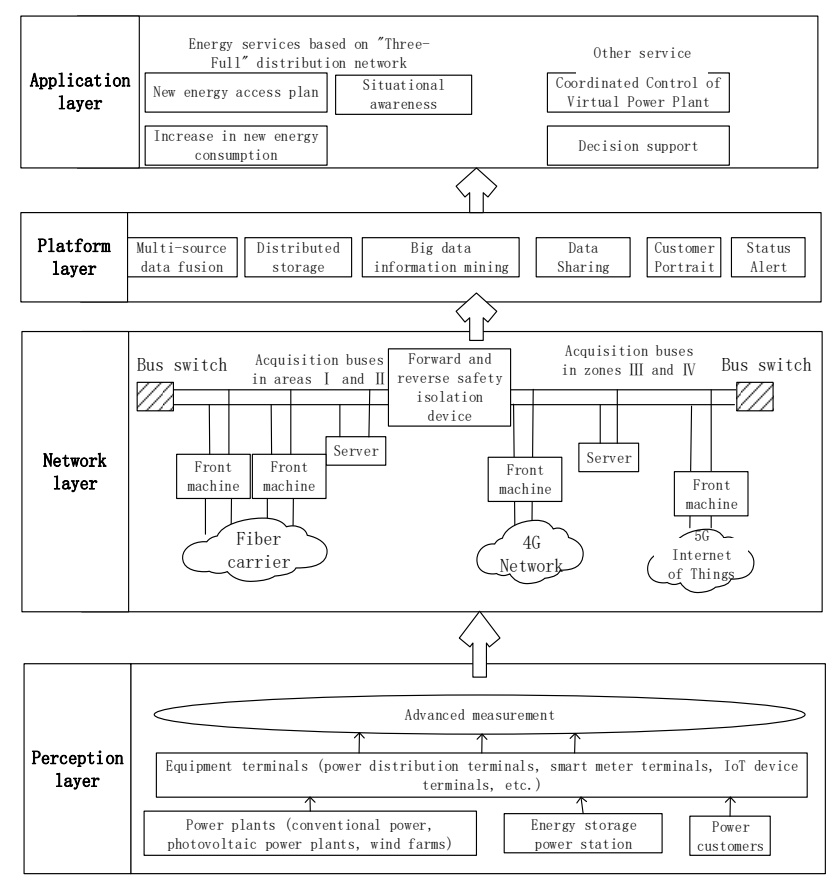

Fig. 4. Platform Architecture Design

The design idea of each layer module is as follows:

\section{Perception layer}

At the perception level, the power generation and operating status of new energy sources, the Ally electric operating parameters of energy storage power stations, and the electricity consumption load of customers using electricity are Ally aware of their equipment terminals through an advanced measurement system, thereby providing a basis for the upper layer data.

\section{Network layer}

The platform layer uses fiber carrier communication in Zones $1 / 2$. In addition to using the existing $4 \mathrm{G}$ information transmission network in Zones 3/4, in order to meet the "ultra-high bandwidth and ultra-low "Time delay, ultra-large-scale connection" requirements, and 5G-based IoT communication technology is also used to transmit the measurement data of the perception layer to the platform layer. The basic data is connected to the platform layer through the integrated data access interface using the communication network.

\section{Platform layer}

The cloud computing platform processes network layer data and provides a series of services. The service content not only includes data services such as multisource data fusion, distributed storage, big data information mining, data resource sharing, etc., but also can provide grid business services such as customer portraits, power grid security status early warning, etc.

\section{Application layer}

In order to facilitate the connection of new energy to the power grid, this layer provides access planning functions and provides access solutions for new energy power plants. Including new energy access capacity, access location, access specification requirements, etc; "TripleAlls" distribution network contains many new energy power sources, controllable loads and energy storage equipment. In order to monitor the operating status of the entire power grid, this layer provides With the situational awareness function, it is possible to understand "what is going on" (potential situational awareness), "why happening" (potential situational understanding) and "what will happen" (potential situation prediction) of the "Triple-Alls" distribution network.

In addition, the "Triple-Alls" distribution network platform achieves the purpose of increasing the proportion of new energy consumption through coordinated control measures of virtual power plants.

\section{Application functions of "triple-alls" distribution network}

\section{1 " Triple-alls " distribution network "all access" services}

The "All access" service of the "Triple-Alls" distribution network is reflected in its new energy power plant access planning function. The new energy power plant access grid planning includes: determining the way of new energy grid connection, grid voltage level, grid point distribution, Functions such as grid connection capacity.

In addition to the centralized power generation plan, the distributed new energy plan follows the principles of clean and efficient, decentralized layout, and nearby use. Installed on the demand side, the operation mode is mainly spontaneous and self-use, and the excess power is connected to the Internet.

\section{2 "Triple-alls" distribution network "all sensing" service}

The "All sensing" service of the "Triple-Alls" distribution network includes three different functions: situational awareness, situational understanding, and situational prediction.

Situational awareness: Through the data provided by the "Triple-Alls" distribution network platform layer, 
it can sense the important characteristics of new energy power and user load changes, so as to provide support for situational understanding and prediction;
Situation
understanding:
Analyse the characteristics of power and load changes detected by the situation, and obtain the relevant rows between the "Triple-Alls" distribution network operation status and historical status information and measurement information.

Situation prediction: Based on the results of awareness and understanding of the situation, aiming at the uncertainty changes of the "Triple-Alls" distribution network, the safety risk assessment is carried out through the power flow calculation and the state assessment technology, and the safety prediction of the operation status of the power grid is performed. At the same time, in order to reduce the pressure on the power balance of the power grid, the application function also makes a precise prediction of the output and load characteristics of the new energy power generation unit.

At the same time, in order to ensure the safe, stable, economical, and flexible operation of the "Triple-Alls" distribution network, the distribution network system provides a virtual power plant coordinated control function and auxiliary decision-making functions. The platform uses the results of situational awareness to realize the balance between power generation and load through distributed network-source coordination control technology, thereby ensuring the safe and economic operation of the grid operation. When uncertain events such as grid failure occur, the system Status warning content, combined with situational awareness results, to assist staff in decision-making.

\section{3 "Triple-alls" distribution network "all consumption" service}

The "All consumption" service provides suggestions for improving the consumption rate through coordinated control strategies. The coordinated control strategy needs to give All play to the complementarity of photovoltaic power generation, wind power generation, and energy storage device operation, and use the charge and discharge of energy storage devices to smooth wind power and solar power output, reduce its volatility, and improve the security and stability of grid operation. In addition, in the control process, the advantages of flexible load utilization can be Ally utilized, and the "source-grid-charge" comprehensive coordination is used to increase the consumption rate of renewable energy.

\section{Summary}

This article aims at the "Triple-Alls" distribution network. First, it analyses the characteristics and needs of key technologies compared with traditional distribution networks. It explains the ubiquitous IoT technology, advanced communication technology, virtual power plant technology, and cloud computing technology. The importance of the "Triple-Alls" distribution network; then the "Triple-Alls" distribution network information model is constructed. The model focuses on extending the power generation model and the power load model. Compared with the traditional distribution network model, the content is more abundant. Next, the "Triple-Alls" distribution network platform architecture was designed. Although the platform follows the traditional "sensing layer-network layerplatform layer-application layer" structure, All consideration has been given to the "Triple-Alls" characteristics of the distribution network. In order to improve the perception level of the entire network, IoT terminal equipment is set up at the perception layer. The application of $5 \mathrm{G}$ communication technology has increased the speed of data transmission and the perception speed has been improved. The "new energy power plant access planning function" in the application layer helps to improve the level of new energy power supply access, and the "new energy consumption proportion increase" function is based on the "netsource-load" coordinated control based on the virtual power plant To improve the level of consumption of new energy. Finally, the article elaborates on the implementation of functions such as "new energy plant planning", "potential situation awareness", and "new energy consumption increase" of the "Triple-Alls" distribution network.

\section{Acknowledgement}

The project research was strongly supported by the company's colleagues, Huzhou Electric Power Company and Huzhou Electric Power Design Institute staff. So I would give sincere thanks for all the friends. Project Number is 2019-HUZJTKJ-27.

\section{References}

1. Xu Peng, Sun Yi, Shi Kun, et al. Interface design of electricity information collection system and business application platform [J]. China Electric Power, 49, pp.136-140 (2016)

2. Shen Bo, Cai Zexiang, Dai Guanquan, et al. Communication analysis of intelligent power distribution information collection service for ubiquitous electric power Internet of Things [J]. Electric Power Construction, 40,pp.27-34(2019)

3. Sun Zhandong, Jin Youhao, Topology Research of Ubiquitous Electricity IoT Distribution Network [J]. Rural Electrification, 8 ,pp.26-29(2019)

4. Li Bingsen, Hu Quangui, Chen Xiaofeng, et al. Research and design of data center in power grid enterprises [J]. Electric Power Information and Communication Technology, 17, pp.29-34(2019)

5. Sun Zhandong, Jin Youhao, Topology Research of Ubiquitous Electricity IoT Distribution Network [J]. Rural Electrification, 387,pp.26-29(2019)

6. Ge Leiyu, Key technologies and evaluation methods of smart power distribution cloud platform with 
distributed power [D]. Tianjin: Tianjin University, (2016)

7. Wu Shan, Yang Lixing, Wang Zeren, et al. Research status and analysis of public information model for power grids [J]. Electrical Engineering, 2 ,pp.16(2017)

8. Wang Zhiying, Zhang Shijun, Deng Yi, Research and design of data center in power grid enterprises [J]. Automation of Electric Power Systems, 38 ,pp. 29-34(2014)

9. Tao Shun, Zheng Jiawei, Chen Meng, et al. Extension of public information model for active power distribution network [J]. Modern Electric Power, 33,pp.74-80(2016)

10. Yang Yisheng, Zhang Jian, Feng Tiantian. Study on compensation mechanism for peak-regulating ancillary service of nuclear units $[\mathrm{J}]$. Power System Technology,41,pp.2131-2137(2017)
11. Kou Wei. The company Ally deployed the ubiquitous electric power Internet of Things [EB / OL]. Website of State Grid Corporation of China, [2019-04-21]. Http://www.sgcc.com.cn/ html / sgcc_main / co12017021449 / 2019-03 / 09 / 20190309185902970893183_1.shtml

12. Ai Yi, Virtual Power Plant_The Ultimate Configuration of Energy Internet [M]. Science Press, Beijing, (2018)

13. Application integration at electric utilities-Systeminterfaces for distribution management-part11: Common information model (CIM) extensions for distribution (IEC6168-11: 2010) [S]. IEC, (2010)

14. Application integration at electric utilities-Systeminterfaces for distribution management-part13: CIM RDF Model exchange format for distribution (IEC61968-13: 2008) [S]. IEC, (2008)

15. Yang Ming, Research on IEC61968 extended CIM model for distributed power generation information [D]. Changsha: Hunan University, (2017) 